\title{
Analysis of angiographic characteristics of kaposiform hemangioendothelioma and investigation of the value of transcatheter arterial embolization therapy
}

\author{
Xiaoyun Tan ${ }^{1,2}$, Zhenyin Liu ${ }^{1,2}$, Shaoyi Zhou ${ }^{1,2}$, Kunshan Chen ${ }^{1,2}$, Ming Zhang ${ }^{1,2}$, Jiejun Xia ${ }^{1,2}$, \\ Yiqun Guo ${ }^{1,2}$, Zijun Zhou ${ }^{1,2}$ \\ ${ }^{1}$ Guangzhou Women and Children's Medical Center, Guangzhou Medical University, Guangzhou, China; ${ }^{2}$ Department of Interventional Therapy \\ and Vascular Anomalies, Guangzhou Women and Children's Medical Center, Guangzhou, China \\ Contributions: (I) Conception and design: X Tan, Z Liu; (II) Administrative support: Z Liu; (III) Provision of study materials or patients: X Tan, S \\ Zhou; K Chen, J Xia; (IV) Collection and assembly of data: X Tan, M Zhang, J Xia; (V) Data analysis and interpretation: X Tan, Y Guo, Z Zhou, Z \\ Liu; (VI) Manuscript writing: All authors; (VII) Final approval of manuscript: All authors. \\ Correspondence to: Zhenyin Liu. Guangzhou Women and Children's Medical Center, Guangzhou Medical University, Guangzhou, China. \\ Email: gentleman595@163.com.
}

Background: This study aimed to analyze the angiographic characteristics of kaposiform hemangioendothelioma (KHE) and investigate the value of transcatheter arterial embolization (TAE) therapy.

Methods: The clinical data of infants diagnosed with KHE at the department from June 2013 to June 2020 were retrospectively analyzed. Of these, 34 infants received TAE therapy. The efficacy of the treatment was evaluated 4 weeks after the therapy. The angiographic characteristics were analyzed by comparing them with the angiographic characteristics of infantile hemangioma (IH), and the times of TAE therapy and the platelet level after each TAE therapy in infants with KHE were summarized.

Results: The present study showed that the capillary blush of KHE was irregular with an obscure boundary and nonuniform distribution. Many fine feeding arteries were present. The diameter of the feeding arteries was disproportionate to the volume of the tumor blush. The normal arteries were usually embedded in the tumor blush. The angiography of common IH in infants also showed tumor blush, but it was usually round with a clear boundary and uniform staining, and was distributed on 1 side of the normal arterial trunk. The infants with KHE received TAE therapy for 2 to 5 times/case, with a total of 104.0 times, with an average of $3.1 \pm 0.8$ /case. Among which, the platelets continued to decline for 9 times after TAE therapy and the platelets increased to $\geq 100 \times 10^{9} / \mathrm{L}$ in $7.8 \pm 3.2$ days for 95 times after TAE therapy, The average relapse time was $30.0 \pm 15.9$ days.

Conclusions: The feeding arteries of KHE were numerous and fine and were not easily embolized. The application of TAE may rapidly improve the platelet level, but the long-term effect is poor.

Keywords: Angiography; embolization; hemangioma; kaposiform hemangioendothelioma (KHE); KasabachMerritt phenomenon (KMP)

Submitted Sep 18, 2021. Accepted for publication Nov 03, 2021.

doi: $10.21037 / \mathrm{tp}-21-486$

View this article at: https://dx.doi.org/10.21037/tp-21-486 


\section{Introduction}

In 1997, Enjolras (1) and Sarkar (2) proposed that the vascular tumor associated with the KasabachMerritt phenomenon (KMP) was actually kaposiform hemangioendothelioma (KHE) or tufted angioma (TA), rather than infantile hemangioma (IH), which was then adopted by the International Society for the Study Vascular Anomalies (ISSVA) (3). KHE has intermediate tumor type with locally aggressive characteristics and many reports have suggested that $\mathrm{KHE}$ was different from $\mathrm{IH}$ in clinical presentation, biological behavior, treatment, and prognosis (4-9). However, rare research reported the angiographic characteristics of KHE. This study found that the angiographic characteristics of KHE were also completely different from those of IH and the value of transcatheter arterial embolization (TAE) for KHE was also investigated.

We present the following article in accordance with the STROBE reporting checklist (available at https://dx.doi. org/10.21037/tp-21-486).

\section{Methods}

This retrospective study was conducted in accordance with the Declaration of Helsinki (as revised in 2013) and approved by the Ethics Committee at Guangzhou Women and Children's Medical Center (No. 2016061605). Informed consent was taken from all the patients' guardians.

\section{Clinical data}

The clinical data of children diagnosed with $\mathrm{KHE}$ at the department from June 2010 to June 2017 were retrospectively analyzed. Of these, 34 cases received TAE therapy, including 18 males and 16 females. The age of infants who received the first TAE therapy was 232 months, with an average of $8.2 \pm 6.6$ months. The foci were located on the body surface in 34 cases ( 9 cases, upper limb; 7 cases, lower limb; 6 cases, maxillofacial region; 5 cases, chest wall; 4 cases, neck; 2 cases, abdominal wall; and 1 case, occipital region). The tumor enlarged rapidly in all infants and spread to surrounding areas within 1 week. The surface was dark red in color, local skin temperature increased, and ecchymosis occurred. The results of ultrasound examination showed a hypoechoic mass with an obscure boundary and a rich blood supply in the mass. All cases were reviewed by the interdisciplinary team, and a consensus was reached on the diagnosis of KMP. The diagnosis was based on a review of the digital photographs, imaging, clinical history, and laboratory data. All children had previously been treated with corticosteroids, sclerotherapy, blood transfusion, or compression therapy with a bandage; however, these treatments had been ineffective. At the time of admission, the platelet count was $(8.0-75.0) \times 10^{9} / \mathrm{L}$, with an average of $(42.4 \pm 17.9) \times 10^{9} / \mathrm{L}$ (Table 1$)$.

\section{Methodology}

The clinical data of 34 infants diagnosed with KHE and the angiographic data of 48 infants with $\mathrm{IH}$ in the hospital were retrospectively analyzed. The TAE treatment of $\mathrm{KHE}$ was as follows (10): corticosteroids (dexamethasone, $1-2 \mathrm{mg} / \mathrm{kg} / \mathrm{d}$, intravenous drip) or platelet $(10-15 \mathrm{~mL} / \mathrm{kg})$ infusion was given before TAE procedures. After the platelet count increased to $50 \times 10^{9} / \mathrm{L}$ or above, TAE was performed immediately. A 4.0-F pediatric puncture sheath kit was used to puncture the right or left femoral artery using the Seldinger technique under general anesthesia with tracheal intubation. A 4.0-F Cobra catheter (Terumo, Tokyo, Japan) was placed into the focus for angiography, and the angiographic characteristics were carefully assessed. Through the 4.0-F catheter, a 2.7-F Progreat catheter (Terumo Corp., Japan) or 1.7-F nerve microcatheter (EV3 Inc., Plymouth, MN, USA) was super-selectively introduced and directed to the feeding artery. Then, iodinated oil emulsion was injected into the feeding artery of the tumor followed by materials using polyvinyl alcohol particles (350$560 \mu \mathrm{m}$, Cook Medical, Inc., Bloomington, IN, USA) or absolute ethanol (10). The iodinated oil emulsion consisted of bleomycin A5 (Harbin Laiboten Pharmaceutical Co., Ltd., Harbin, China) as a sclerosant, iodinated oil (Lipiodol; Laboratoire Guerbet, Roissy-Charles de Gaulle, France) as an embolization agent, dexamethasone (Shandong Lukang Cisen Pharmaceutical Co., Ltd., Shandong, China), and a contrast agent (Omnipaque; GE Healthcare, Shanghai, China). The overall volume of this emulsion was $6-10 \mathrm{~mL}$, and the concentration of the iodinated oil was $20-33 \%$. The corticosteroids and other treatments were stopped after TAE. The therapeutic effect was evaluated 4 weeks later. The aforementioned treatment was repeated if the platelet levels were still $<100 \times 10^{9} / \mathrm{L}$. The angiographic characteristics of 34 cases with $\mathrm{KHE}$ and 48 cases with $\mathrm{IH}$ were analyzed by 3 interventional physicians together.

The criteria of efficacy were as follows: (I) cured: tumor disappeared, platelet count returned to the normal level, 
Table 1 General data of 34 cases with KHE and 48 cases with IH

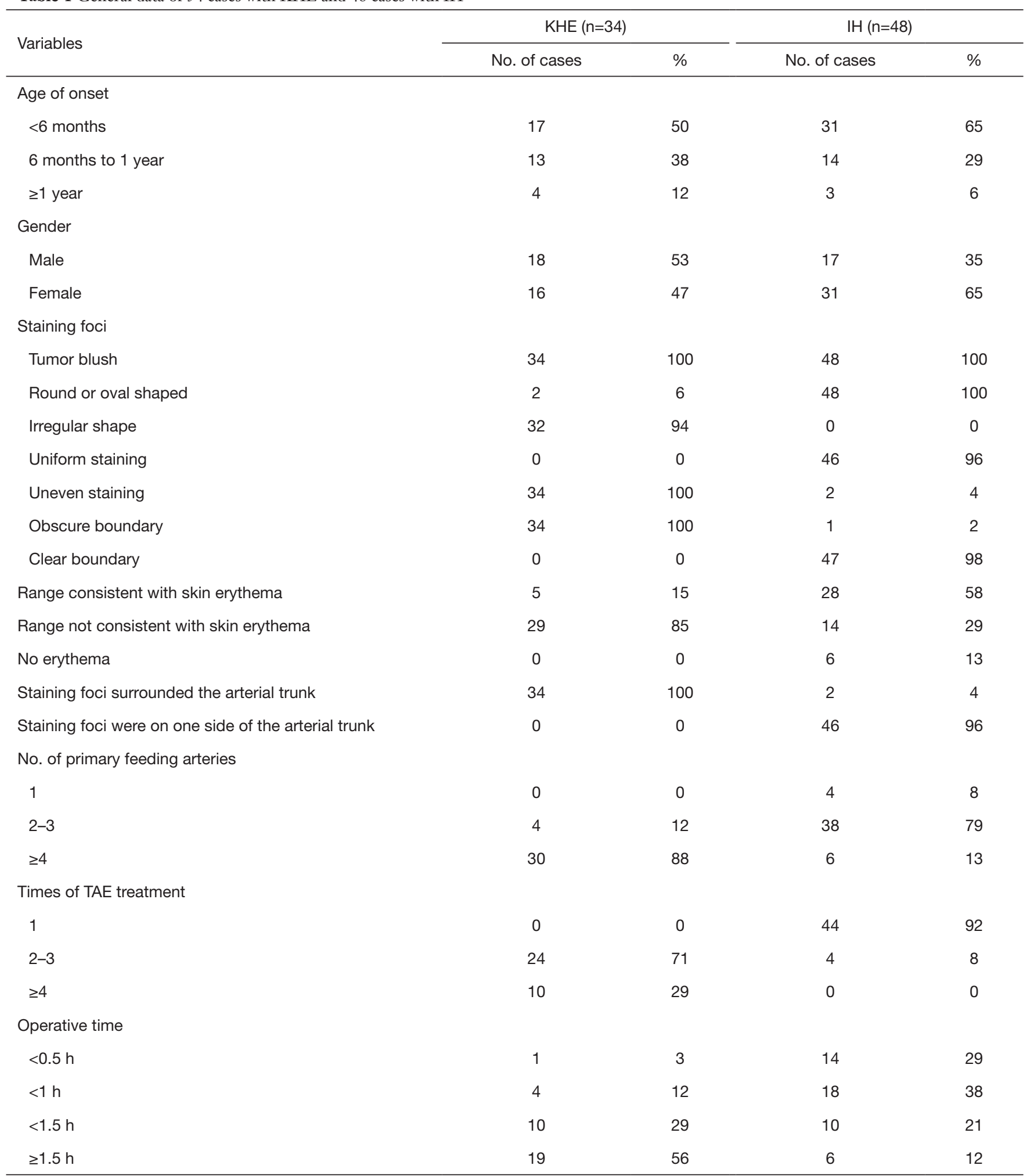

$\mathrm{IH}$, infantile hemangioma; h, hour; KHE, kaposiform hemangioendothelioma; TAE, transcatheter arterial embolization. 


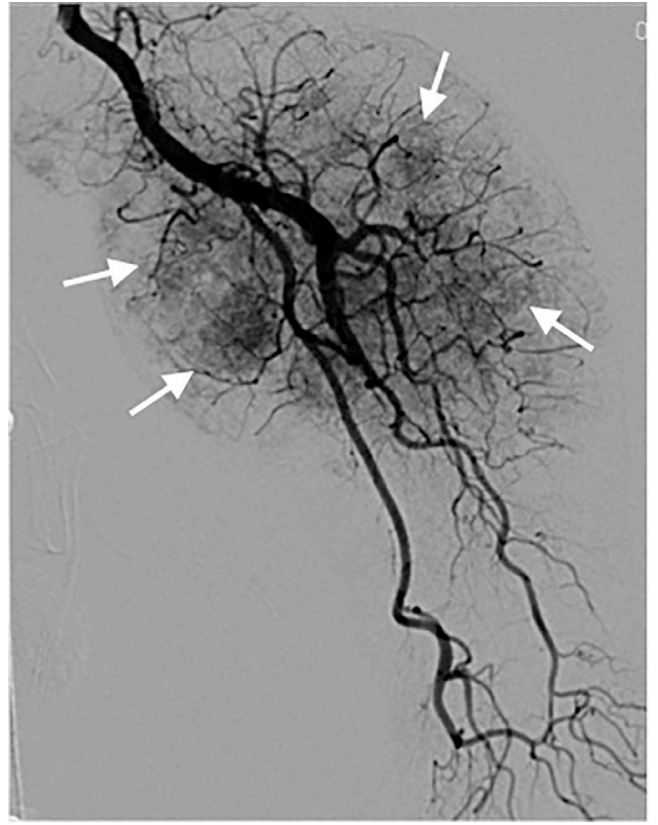

Figure 1 Typical angiographic characteristics of KHE in left upper extremity: tumoral staining focus with irregular morphology (white arrow), uneven staining, obscure boundary, the staining focus surrounding the normal arterial trunk, numerous fine feeding arteries, and the diameter not proportional to the size of tumor. KHE, kaposiform hemangioendothelioma.

and the disease did not recur during the 6-month followup or longer; (II) improved: the volume of tumor decreased, and the platelet count obviously rebounded; (III) ineffective: the volume of tumor continued to increase, and the platelet count did not rebound; and (IV) dead.

\section{Statistical analysis}

SPSS for Windows version 16.0 (SPSS, Inc., Chicago, Illinois, USA) was used for all statistical analyses. The means of the quantitative data were compared with a onesample Kolmogorov-Smirnov test (a normality test).

\section{Results}

The capillary blush of KHE was irregular with an obscure boundary and nonuniform distribution. A number of fine feeding arteries were present. The diameter of the feeding arteries was disproportionate to the volume of the tumor blush. The normal arteries were usually embedded in the

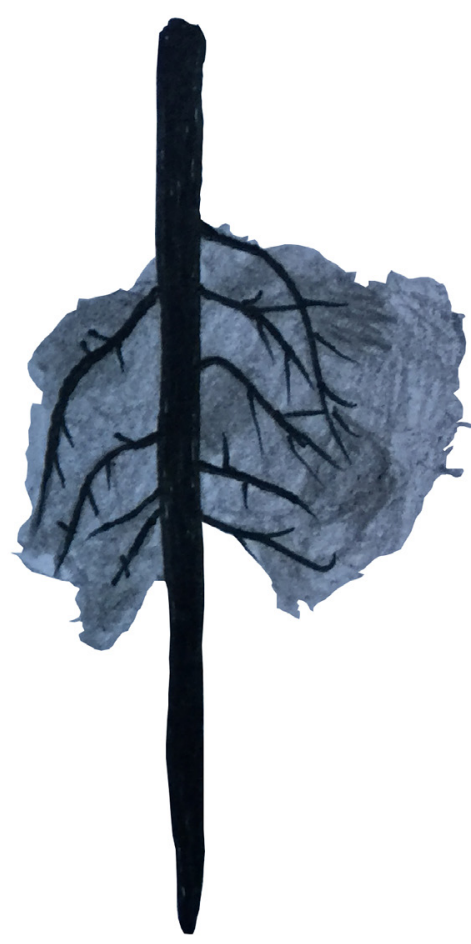

Figure 2 Angiographic characteristics pattern of KHE. KHE, kaposiform hemangioendothelioma.

tumor blush (Figures 1,2). The angiography of common $\mathrm{IH}$ in infants also showed tumor blush, but it was usually round with a clear boundary and uniform staining, and was distributed on a unilateral side of the normal arterial trunk. Usually, 1-4 feeding arteries were present, and the diameter was proportional to spread the tumor size (Figures 3,4). More details are shown in Table 1.

The infants with KHE received angiography and TAE therapy 2-5 times/case, with a total of 104 times. Among Which, the platelets continued to decline for 9 times after TAE therapy and the platelets increased to $\geq 100 \times 10^{9} / \mathrm{L}$ in $7.8 \pm 3.2$ days for 95 times after TAE therapy. The platelet level fluctuated thereafter, and the average relapse time was $30.0 \pm 15.9$ days. The operative time in $85 \%(29 / 34)$ of cases with $\mathrm{KHE}$ was more than $1 \mathrm{~h}$. The operative time of $\mathrm{IH}$ was also more than $1 \mathrm{~h}$, accounting for only $33 \%(16 / 48)$. All infants were followed up from 6 months to 2 years and finally received other therapies, such as corticosteroids, vincristine, or sirolimus. A total of 6 children were cured, and 28 children improved. 


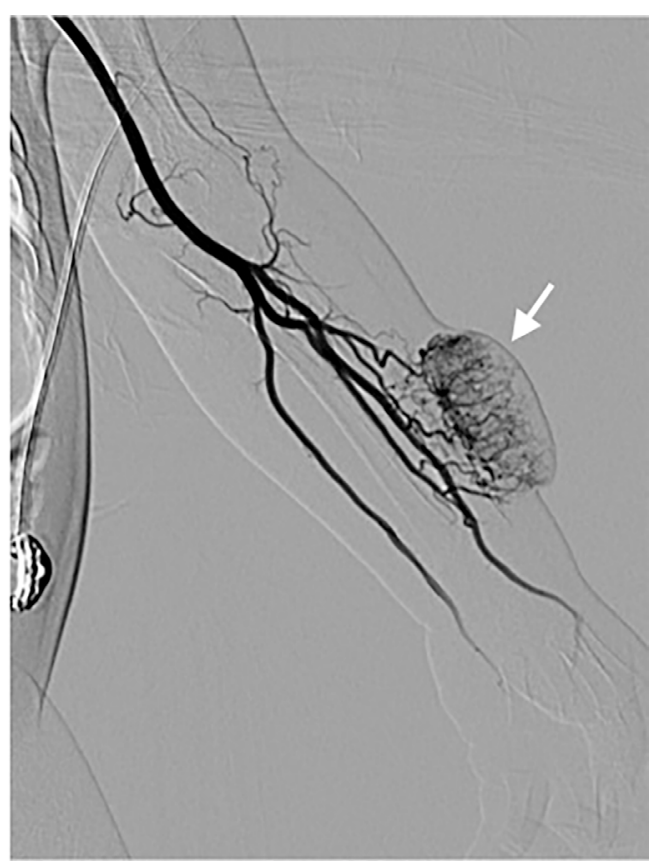

Figure 3 Typical angiographic characteristics of IH in left upper extremity: round tumoral staining focus, clear boundary, uniform staining, distributed on one side of the normal arterial trunk (white arrow), usually $1-4$ feeding arteries, and the diameter proportional to the size of tumor. IH, infantile hemangioma.

\section{Discussion}

The rare borderline tumor of KHE is predominantly associated with KMP. It occurs in the neonatal or infantile period, with a decline in platelet count, hypofibrinoemia, and blood in the low coagulation state, followed by bleeding and anemia. The severity of the disease leads to systemic disseminated intravascular coagulation, which is a serious threat to life. The mortality rate is as high as $12-30 \%$, and the clinical treatment of $\mathrm{KHE}$ is quite challenging (4). The imaging investigations of $\mathrm{KHE}$ mainly include ultrasound, computed tomography (CT), and magnetic resonance imaging (MRI), which show that the blood supply is rich, and the density, echo, or signal is uneven (11). However, the angiographic characteristics of KHE have rarely been reported. Although a few studies have mentioned that the feeding arteries of KHE are obviously fine (10,12-14), larger case studies have not yet been reported. The present study showed that the imaging characteristics of KHE were completely different from those of IH according to the analysis of angiographic manifestations of larger cases with KHE and IH, with the

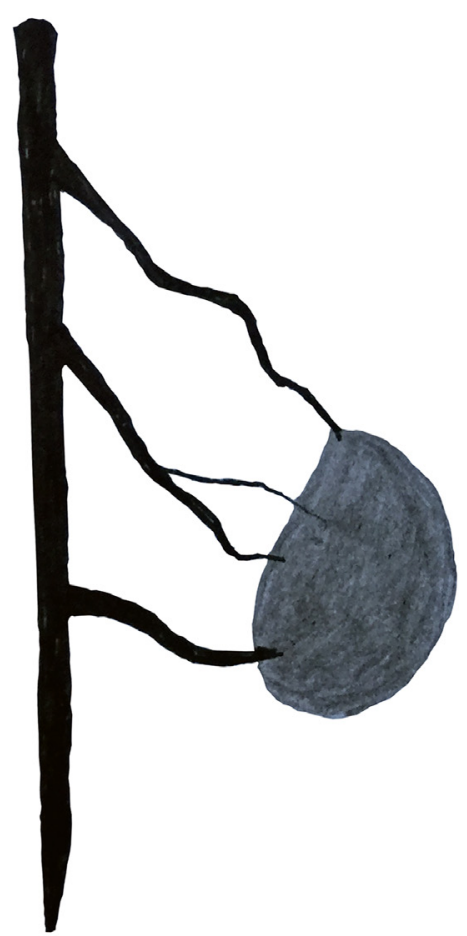

Figure 4 Angiographic characteristics pattern of IH. IH, infantile hemangioma.

following implications: (I) because the feeding arteries of KHE were fine and numerous, the TAE procedure was different from that of $\mathrm{IH}$; it required the embolization of more blood vessels and the use of a thinner microcatheter. Therefore, the operation was more time-consuming. In this study, the operative time in $85 \%$ (29/34) of KHE cases was more than $1 \mathrm{~h}$. The operative time of $\mathrm{IH}$ was also more than $1 \mathrm{~h}$, accounting for only $33 \%$ (16/48). In addition, a $2.7-\mathrm{F}$ microcatheter was often used in this study for superselective embolization in treating IH, but it was difficult to embolize KHE completely due to reflux, which might be one of the most important reasons for recurrence. In this study, 71\% (24/34) of cases with KHE received TAE therapy 2 or 3 times, and 29\% (10/34) of cases received the treatment 4 or more times. The authors suggested using a 1.7-F microcatheter for the superselective embolization of thinner feeding arteries. Absolute ethanol was injected to perform sclerotherapy through the 1.7-F microcatheter, but the dose was limited strictly because of the risk of skin or tumor necrosis. It was suggested that the operation should be performed by experienced interventional radiologists. (II) The feeding arteries of KHE were not 
proportional to the surrounding normal vascular trunk and tumor size. The thin and rotating feeding arteries were directly isolated from great vessels, and the blood flowed directly from great arterial vessels into abruptly narrowed and thin feeding arteries. Therefore, surge flow occurred easily, resulting in platelet obstruction. However, the vessels of IH were bifurcated, and the blood flow was normal laminar flow, which could not block the platelets. This characteristic of blood flow was consistent with the findings of related pathological studies (15). (III) The tumor blush border of KHE was unclear and uneven. It usually surrounded the normal arterial trunk, indicating that the true location of KHE focus was deep and often invaded the vascular trunk, which had the biological behavior of malignant tumors. Therefore, it was defined as a borderline tumor by ISSVA. Of course, this added great difficulty and risk to the surgical operation. If the excision was not completely performed, it led to the recurrence of foci; therefore, this study did not recommend that surgical resection be considered as the preferred therapeutic option. In fact, for inoperable and critical cases, TAE therapy could temporarily reduce foci and improve the platelet level. In this study, the platelet count of infants increased to $\geq 100 \times 10^{9} / \mathrm{L}$ in $7.8 \pm 3.2$ days, indicating that TAE therapy took effect quickly and provided the conditions for further operation or drug treatment for infants with KHE. (IV) In some developing countries, KHE without KMP has been shown as prone to misdiagnosis as IH, and TAE is still widely used in treating giant IH. The authors suggest that the typical angiographic characteristics of KHE are useful for their further identification. Besides, biopsy is gold standard for diagnosis and should be performed if possible and safe. ECs in IH lesions are positive for Glut-1, which is absent in KHE and other vascular tumors $(16,17)$.

At present, various treatments are available for KHE, but none of these treatments have been uniformly effective. Complete surgical resection is often not a viable option because most KHE are infiltrative in nature and often invade large neurovascular structures, muscle, and fascia. Moreover, as tumors may occur in a discontinuous fashion, clear surgical margins are nearly impossible to achieve. For cases of KHE associated with KMP, firstline therapy with intravenous vincristine $0.05 \mathrm{mg} / \mathrm{kg}$ once weekly and oral prednisolone $2 \mathrm{mg} / \mathrm{kg} / \mathrm{d}$ or intravenous methylprednisolone $1.6 \mathrm{mg} / \mathrm{kg} / \mathrm{d}$ is recommended by the consensus-derived practice standards (5). For cases of KHE that require intervention because of growth or symptoms but do not have KMP, oral prednisolone $2 \mathrm{mg} / \mathrm{kg} / \mathrm{d}$ is the recommended first-line therapy (5). However, these recommendations are based on expert opinion rather than rigorous clinical studies. A prospective study of sirolimus as treatment for complicated vascular anomalies at the Cincinnati Children's Hospital Medical Center described sirolimus as an effective treatment therapy (18). Embolization therapy initially used as an adjuvant therapy with steroids, is emerging as another treatment modality (19). Patients with KMP should be treated aggressively with a combined regimen and monotherapy is usually not recommended (9). The application of combination treatment often leads to a difficult comparison of various treatment plans. In this study, the infants received TAE therapy 2-5 times/case. The platelet count increased to $\geq 100 \times 10^{9} / \mathrm{L}$ in $7.8 \pm 3.2$ days after the TAE procedure, indicating that the TAE therapy could rapidly improve the platelet count. Unfortunately, relapse occurred in $30.9 \pm 15.9$ days after the TAE procedure. The platelet count continued to decline by 9 times after TAE therapy, which might be related to numerous, fine tumor-feeding arteries, and neovascularization after TAE therapy. More detailed reasons require further elucidation.

Of course, this study had some limitations. The efficacy of TAE might have been affected by different operators and the location of focus. It's reported that the risk of KHE increases dramatically when tumor infiltrates muscle or when KHE arises in the retroperitoneum or mediastinum (4). Receiving various treatments before TAE could also affect the assessment of efficacy. Moreover, the roles of TAE in KHE for various comprehensive treatments were not further studied. Multicenter prospective clinical trials with a larger sample size and longer follow-up period are required. However, it was interpreted that the angiographic characteristics of KHE differed from those of common $\mathrm{IH}$, and the feeding arteries of KHE were numerous and fine. Interventional radiologists should fully understand these differences before operation and make adequate preparations.

\section{Acknowledgments}

Funding: This study was supported by Guangzhou Health Science and Technology Project (No. 20201A011031) and the Project of Guangzhou Women and Children's Medical Center (No. IP-2019-015). 


\section{Footnote}

Reporting Checklist: The authors have completed the STROBE reporting checklist. Available at https://dx.doi. org/10.21037/tp-21-486

Data Sharing Statement: Available at https://dx.doi. org/10.21037/tp-21-486

Conflicts of Interest: All authors have completed the ICMJE uniform disclosure form (available at https://dx.doi. org/10.21037/tp-21-486). The authors have no conflicts of interest to declare.

Ethical Statement: The authors are accountable for all aspects of the work in ensuring that questions related to the accuracy or integrity of any part of the work are appropriately investigated and resolved. This retrospective study was conducted in accordance with the Declaration of Helsinki (as revised in 2013) and approved by the Ethics Committee at Guangzhou Women and Children's Medical Center (No. 2016061605). Informed consent was taken from all the patients' guardians.

Open Access Statement: This is an Open Access article distributed in accordance with the Creative Commons Attribution-NonCommercial-NoDerivs 4.0 International License (CC BY-NC-ND 4.0), which permits the noncommercial replication and distribution of the article with the strict proviso that no changes or edits are made and the original work is properly cited (including links to both the formal publication through the relevant DOI and the license). See: https://creativecommons.org/licenses/by-nc-nd/4.0/.

\section{References}

1. Enjolras O, Wassef M, Mazoyer E, et al. Infants with Kasabach-Merritt syndrome do not have "true" hemangiomas. J Pediatr 1997;130:631-40.

2. Sarkar M, Mulliken JB, Kozakewich HP, et al. Thrombocytopenic coagulopathy (KasabachMerritt phenomenon) is associated with Kaposiform hemangioendothelioma and not with common infantile hemangioma. Plast Reconstr Surg 1997;100:1377-86.

3. Dasgupta R, Fishman SJ. ISSVA classification. Semin Pediatr Surg 2014;23:158-61.

4. Croteau SE, Liang MG, Kozakewich HP, et al. Kaposiform hemangioendothelioma: atypical features and risks of
Kasabach-Merritt phenomenon in 107 referrals. J Pediatr 2013;162:142-7.

5. Drolet BA, Trenor CC 3rd, Brandão LR, et al. Consensusderived practice standards plan for complicated Kaposiform hemangioendothelioma. J Pediatr 2013;163:285-91.

6. Yasui N, Koh K, Kato M, et al. Kasabach-Merritt phenomenon: a report of 11 cases from a single institution. J Pediatr Hematol Oncol 2013;35:554-8.

7. Wang Z, Li K, Yao W, et al. Steroid-resistant kaposiform hemangioendothelioma: a retrospective study of 37 patients treated with vincristine and long-term follow-up. Pediatr Blood Cancer 2015;62:577-80.

8. Wong BL, Lee VN, Tikka T, et al. Kaposiform haemangioendothelioma of the head and neck. Crit Rev Oncol Hematol 2016;104:156-68.

9. Ji Y, Chen S, Yang K, et al. Kaposiform hemangioendothelioma: current knowledge and future perspectives. Orphanet J Rare Dis 2020;15:39.

10. Tan X, Chen M, Zhang J, et al. Treatment of Corticosteroid-Resistant Vascular Tumors Associated with the Kasabach-Merritt Phenomenon in Infants: An Approach with Transcatheter Arterial Embolization Plus Vincristine Therapy. J Vasc Interv Radiol 2016;27:569-75.

11. Ryu YJ, Choi YH, Cheon JE, et al. Imaging findings of Kaposiform Hemangioendothelioma in children. Eur J Radiol 2017;86:198-205.

12. Nakib G, Calcaterra V, Quaretti P, et al. Chemotherapy and surgical approach with repeated endovascular embolizations: safe interdisciplinary treatment for kasabach-merritt syndrome in a small baby. Case Rep Oncol 2014;7:23-8.

13. Zhou SY, Li HB, Mao YM, et al. Successful treatment of Kasabach-Merritt syndrome with transarterial embolization and corticosteroids. J Pediatr Surg 2013;48:673-6.

14. Garcia-Monaco R, Giachetti A, Peralta O, et al. Kaposiform hemangioendothelioma with KasabachMerritt phenomenon: successful treatment with embolization and vincristine in two newborns. J Vasc Interv Radiol 2012;23:417-22.

15. Lyons LL, North PE, Mac-Moune Lai F, et al. Kaposiform hemangioendothelioma: a study of 33 cases emphasizing its pathologic, immunophenotypic, and biologic uniqueness from juvenile hemangioma. Am J Surg Pathol 2004;28:559-68.

16. Krowchuk DP, Frieden IJ, Mancini AJ, et al. Clinical Practice Guideline for the Management of Infantile Hemangiomas. Pediatrics 2019;143:e20183475. 
17. Léauté-Labrèze C, Harper JI, Hoeger PH. Infantile haemangioma. Lancet 2017;390:85-94.

18. Adams DM, Trenor CC 3rd, Hammill AM, et al. Efficacy and Safety of Sirolimus in the Treatment of Complicated Vascular Anomalies. Pediatrics 2016;137:e20153257.

Cite this article as: Tan X, Liu Z, Zhou S, Chen K, Zhang M, Xia J, Guo Y, Zhou Z. Analysis of angiographic characteristics of kaposiform hemangioendothelioma and investigation of the value of transcatheter arterial embolization therapy. Transl Pediatr 2021;10(12):3194-3201. doi: 10.21037/tp-21-486
19. Adams DM, Hammill A. Other vascular tumors. Semin Pediatr Surg 2014;23:173-7.

(English Language Editor: J. Jones) 